07

\title{
Динамика проникающих дислокаций в пористых гетероэпитаксиальных пленках GaN
}

\author{
(C) М.Ю. Гуткин ${ }^{1-3}$, Е.А. Ржавцев ${ }^{1}$ \\ ${ }^{1}$ Институт проблем машиноведения РАН, \\ Санкт-Петербург, Россия \\ ${ }^{2}$ Санкт-Петербургский политехнический университет Петра Великого, \\ Санкт-Петербург, Россия \\ ${ }^{3}$ Санкт-Петербургский национальный исследовательский университет \\ информационных технологий, механики и оптики, \\ Санкт-Петербург, Россия \\ E-mail: m.y.gutkin@gmail.com
}

(Поступила в Редакцию 23 мая 2017 г.)

С помощью компьютерного моделирования методом двумерной дискретной динамики дислокаций исследовано поведение проникающих дислокаций в пористых гетероэпитаксиальных пленках нитрида галлия $(\mathrm{GaN})$. Использована расчетная схема, в которой поры моделировались сечениями цилиндрических полостей, упруго взаимодействующих с однонаправленными параллельными краевыми дислокациями, имитирующими проникающие дислокации. Получены временные зависимости координат и скоростей каждой дислокации из исследованных дислокационных ансамблей. Выполнена визуализации текущей структуры дислокационного ансамбля в виде карты расположения дислокаций в любой момент времени. Показано, что плотность возникающих дислокационных структур существенно зависит от отношения площади поперечного сечения поры к площади области моделирования. В частности, увеличение доли поверхности пор на поверхности слоя до $2 \%$ должно приводить к снижению конечной плотности проникающих дислокаций примерно в 1.5 раза, а увеличение этой доли до $15 \%$ - примерно в 4.5 раза.

Работа выполнена при поддержке Министерства образования и науки Российской Федерации (грант № 3.3194.2017/PCh).

DOI: $10.21883 /$ FTT.2017.12.45233.164

\section{1. Введение}

Полупроводниковые гетероструктуры на основе нитрида галлия $(\mathrm{GaN})$, полученные эпитаксиальным ростом, широко используются в современных электронных и оптоэлектронных приборах [1-8]. Качество выращенного кристалла $\mathrm{GaN}$ сильно зависит от выбора подложки. В настоящее время в основном используются подложки, изготовленные из других кристаллов, обладающих решеточным несоответствием относительно кристаллической решетки GaN. Это несоответствие вызывает в растущем слое значительные напряжения несоответствия, релаксация которых сопровождается прорастанием в этот слой проникающих дислокаций (ПД). ПД оказывают существенное негативное воздействие на проводящие и оптоэлектронные свойства гетероструктур, полученных на основе III-нитридных полупроводников $[9,10]$, так что уменьшение их плотности является важной научной и технологической задачей.

Эффективным методом снижения плотности ПД может служить формирование промежуточных пористых слоев $\mathrm{GaN}$, которые затем заращиваются сплошными слоями. Из экспериментов известно, что применение пористых подложек приводит к значительному снижению плотности ПД в растущих пленках $\mathrm{GaN}$ [11-14]. Тем не менее в настоящее время отсутствует пол- ное понимание влияния пористости на эволюцию ПД в заращенном слое $\mathrm{GaN}$. Только в самые последние годы появились первые физические модели, развитые на основе реакционно-кинетической модели поведения дислокаций $[15,16]$ и описывающие эволюцию плотности ПД в таких слоях $[17,18]$.

Описание кинетики эволюции плотности ПД указывает на общую тенденцию к ее снижению с увеличением толщины пористого слоя, однако не может прояснить деталей упругого поведения взаимодействующих между собой ПД вблизи пор. Общее представление об особенностях этого поведения и о микромеханизмах снижения плотности ПД можно получить, используя альтернативный подход, который заключается в компьютерном моделировании дискретной динамики дислокаций вблизи пор в напряженном растущем слое $\mathrm{GaN}$. B разработке этого подхода можно взять за основу результаты, полученные при развитии двух важных направлений в теории дислокаций. Первое направление - это изучение упругого взаимодействия дислокаций с внутренними порами в твердом теле [19-38]. Второе направление это анализ динамики формирования различных дислокационных структур в условиях внешних воздействий в рамках двумерной ньютоновской динамики [39-43]. В последние годы развитие этого направления связано с описанием пластической деформации вблизи 
границ зерен [42,43], клиновых дисклинаций [44-54], трещин [55-59], пор [59-63] и включений [64-66].

Цель настоящей работы - изучение динамики ПД вблизи поры в напряженном слое $\mathrm{GaN}$ с помощью компьютерного моделирования на стыке двух упомянутых выше направлений.

\section{2. Модель}

Рассмотрим модельный случай одномерного решеточного несоответствия слоя и подложки. Пусть в центре области моделирования $\left(-x_{b} \leq x \leq x_{b},-y_{b} \leq y \leq y_{b}\right)$ расположена цилиндрическая пора (рис. 1), имеющая круглое сечение радиуса $R$ с координатами центра, совпадающими $\mathrm{c}$ началом декартовой системы координат $(x=0, y=0)$. Ось поры перпендикулярна поверхности слоя и совпадает с осью $z$. На большом удалении от этой поры, на линии $\left(x=-x_{b}, y\right)$, в момент времени $t=0$ под действием действующего в слое сдвигового напряжения $\tau$, вызванного растягивающим напряжением несоответствия $\sigma$, начинается генерация положительных краевых дислокаций с векторами Бюргерса $\mathbf{b}$, направленными вдоль оси $x$. Одновременно начинается генерация отрицательных дислокаций с векторами Бюргерса - b на линии $\left(x=x_{b}, y\right)$. Распределение дислокаций вдоль оси $y$ и моменты их появления задаются с помощью генератора случайных чисел, а плоскости $y=$ const служат плоскостями скольжения дислокаций. Если в процессе моделирования расстояние между двумя дислокациями противоположных знаков становится меньше $3 a$ ( $a$ - параметр решетки), то считается, что дислокации аннигилируют.

Каждая дислокация в данной модели находится под совместным действием сил, вызванных сдвиговым напряжением $\tau$ и силой изображения со стороны поры. При этом предполагается, что дислокация может перемещаться только в своей плоскости скольжения вдоль

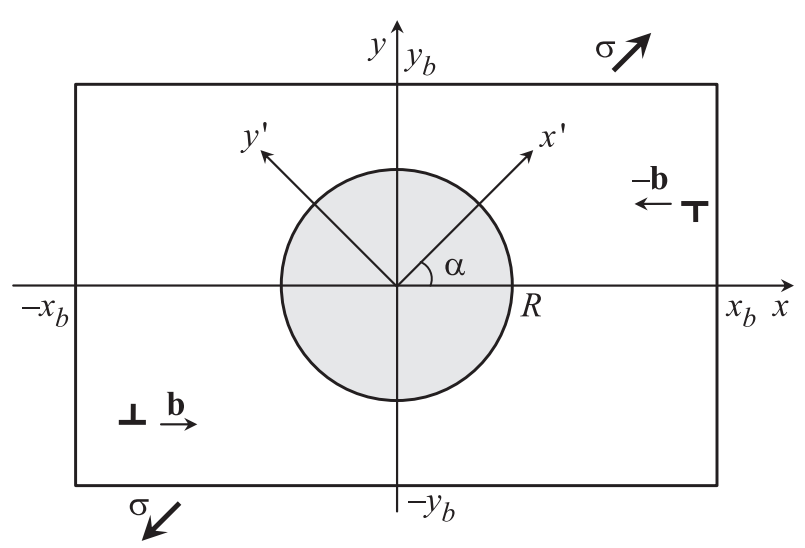

Рис. 1. Начальное состояние области моделирования при $t=0$. Вдали от нее под углом $45^{\circ}$ к оси $x$ приложено однородное растягивающее напряжение $\sigma$, вызванное несоответствием кристаллических решеток слоя и подложки. оси $x$, и в этом случае ее движение определяется только проекциями сил на ось $x$.

Уравнение движения $i$-й дислокации можно представить в виде $[46,47]$

$$
m \frac{d^{2} x_{i}}{d t^{2}}+\beta \frac{d x_{i}}{d t}=F_{i}, \quad i=1,2, \ldots, N
$$

где $m$ - эффективная масса дислокации, $x_{i}-$ eе координата, $\beta-$ коэффициент динамического трения, $F_{i}-$ равнодействующая всех сторонних сил, действующих на дислокацию, $N$ - общее число дислокаций, генерированных за время проведения одного численного эксперимента. Первая производная $d x_{i} / d t$ в этом уравнении учитывает динамическое (вязкое) трение дислокации при ее скольжении в кристаллической решетке. Масса дислокации дана в стандартном приближении [67]: $m=\rho b^{2} / 2$, где $\rho-$ плотность материала, $b-$ величина вектора Бюргерса дислокации.

Суммарную силу, действующую на $i$-ю дислокацию с координатами $\left(x_{i}, y_{i}\right)$, можно записать в следующем виде

$$
F_{i}=F_{\sigma i}+F_{h i}+\sum_{\substack{j=1, j \neq i}}^{N} F_{i j} .
$$

Первое слагаемое в правой части формулы (2) определяет силу, обусловленную приложенным растягивающим напряжением $\sigma$, второе - силу ее упругого взаимодействия с порой (силу изображения), третье - суммарную силу упругого взаимодействия $i$-й дислокации со всеми остальными дислокациями.

Чтобы определить первое слагаемое, воспользуемся готовым решением задачи о влиянии круглого отверстия на распределение напряжений в пластине [68]. Компоненты напряжений в полярной системе координат $(r, \theta)$ :

$$
\begin{gathered}
\sigma_{r r}=\frac{\sigma}{2}\left(1-\frac{R^{2}}{r^{2}}\right)+\frac{\sigma}{2}\left(1-\frac{4 R^{2}}{r^{2}}+\frac{3 R^{4}}{r^{4}}\right) \cos 2 \theta \\
\sigma_{\theta \theta}=\frac{\sigma}{2}\left(1+\frac{R^{2}}{r^{2}}\right)-\frac{\sigma}{2}\left(1+\frac{3 R^{4}}{r^{4}}\right) \cos 2 \theta \\
\sigma_{r \theta}=-\frac{\sigma}{2}\left(1+\frac{2 R^{2}}{r^{2}}-\frac{3 R^{4}}{r^{4}}\right) \sin 2 \theta .
\end{gathered}
$$

Чтобы найти сдвиговое напряжение $\tau$, действующее в плоскостях скольжения дислокаций, перейдем от полярной системы координат к декартовой системе $\left(x^{\prime}, y^{\prime}\right)$, повернутой относительно системы координат $(x, y)$ на угол $\alpha$ (рис. 1). Для этого воспользуемся известными формулами [68]:

$$
\begin{array}{r}
\sigma_{x^{\prime} x^{\prime}}=\sigma_{r r} \cos ^{2} \theta+\sigma_{\theta \theta} \sin ^{2} \theta-2 \sigma_{r \theta} \sin \theta \cos \theta, \\
\sigma_{y^{\prime} y^{\prime}}=\sigma_{r r} \sin ^{2} \theta+\sigma_{\theta \theta} \cos ^{2} \theta+2 \sigma_{r \theta} \sin \theta \cos \theta, \\
\sigma_{x^{\prime} y^{\prime}}=\left(\sigma_{r r}-\sigma_{\theta \theta}\right) \sin \theta \cos \theta+\sigma_{r \theta}\left(\cos ^{2} \theta-\sin ^{2} \theta\right),
\end{array}
$$


где $r^{2}=x^{\prime 2}+y^{\prime 2}, \theta=\arctan \left(x^{\prime} / y^{\prime}\right)$. Выражая $\tau$ через эти компоненты, находим

$$
\tau=-\frac{\sigma_{x^{\prime} x^{\prime}}-\sigma_{y^{\prime} y^{\prime}}}{2} \sin 2 \alpha+\sigma_{x^{\prime} y^{\prime}} \cos 2 \alpha .
$$

Подставляя формулы (3) и (4) в равенство (5), получим для $\alpha=\pi / 4$

$$
\begin{aligned}
\tau= & -\frac{\sigma}{2}\left\{\left(1-\frac{3 R^{2}}{r^{2}}+\frac{3 R^{4}}{r^{4}}\right) \cos ^{2} \theta\right. \\
& \left.+\left(1-\frac{R^{2}}{r^{2}}-\frac{3 R^{4}}{r^{4}}\right) \sin ^{2} \theta\right\} .
\end{aligned}
$$

Тогда первое слагаемое в правой части равенства (2) равно $F_{\sigma i}=b \tau\left(x_{i}, y_{i}\right)$, где $\tau\left(x_{i}, y_{i}\right)$ определяется формулой (6) с учетом того, что $x_{i}=r_{i} \cos \theta_{i}, y_{i}=r_{i} \sin \theta_{i}$ и $r_{i}^{2}=x_{i}^{2}+y_{i}^{2}$, откуда имеем

$$
\begin{aligned}
F_{\sigma i}= & -\frac{b \sigma}{2 r_{i}^{6}}\left\{x_{i}^{2}\left[3 R^{2}\left(R^{2}-r_{i}^{2}\right)+r_{i}^{4}\right]\right. \\
& \left.-y_{i}^{2}\left[R^{2}\left(3 R^{2}+r_{i}^{2}\right)-r_{i}^{4}\right]\right\} .
\end{aligned}
$$

Чтобы найти силу изображения, сначала рассмотрим более общий случай и найдем поля напряжений краевой дислокации в произвольной точке $K$ с учетом длинной цилиндрической поры (рис. 2,a). Решение этой задачи было получено в работе [19] в виде функции напряжений Эри $\psi$, которую можно представить в виде суммы

$$
\psi=\psi_{1}+\psi_{2}
$$

$$
\begin{aligned}
\psi_{1} & =\frac{G b_{x_{0}}}{4 \pi(1-v)} \\
& \times\left\{-2 r_{1} \ln r_{1} \sin \theta_{1}+2\left(r_{2} \ln r_{2} \sin \theta_{2}-r \ln r \sin \theta\right)\right. \\
& \left.-\frac{\left(\xi^{2}-1\right) R}{\xi^{3}}\left[\sin 2 \theta_{2}-\frac{\left(\xi^{2}-1\right) R}{\xi} \frac{\sin \theta_{2}}{r_{2}}\right]-\frac{R^{2} \sin \theta}{r}\right\},
\end{aligned}
$$$$
\psi_{2}=\frac{G b_{y_{0}}}{4 \pi(1-v)}\left\{2 r_{1} \ln r_{1} \sin \theta_{1}-2\left(r_{2} \ln r_{2} \sin \theta_{2}\right.\right.
$$

$$
\begin{aligned}
& -r \ln r \sin \theta)+\frac{\left(\xi^{2}-1\right) R}{\xi^{3}}\left[2 \xi^{2} \ln r_{2}-\cos 2 \theta_{2}\right. \\
& \left.\left.+\frac{\left(\xi^{2}-1\right) R}{\xi} \frac{\cos \theta_{2}}{r_{2}}\right]-2\left(\xi^{2}-1\right) \frac{R}{\xi} \ln r+\frac{R^{2} \cos \theta}{r}\right\},
\end{aligned}
$$

где $G$ - модуль сдвига, $v-$ коэффициент Пуассона, $b_{x_{0}}$ и $b_{y_{0}}-$ величины компонент вектора Бюргерса дислокации в декартовой системе координат $\left(x_{0}, y_{0}, z_{0}\right)$, в которой начало координат лежит в центре поры, ось $z_{0}$ совпадает с осью поры, а ось $x_{0}$ проходит через линию дислокации, ориентированную вдоль оси $z_{0}$ и пересекающую плоскость $\left(x_{0}, y_{0}\right)$ в точке $\left(x_{0}=c, y_{0}=0\right)$ (рис. 2, $a) ; r^{2}=x_{0}^{2}+y_{0}^{2}, r_{1,2}^{2}=x_{01,2}^{2}+y_{0}^{2}, x_{01}=x_{0}-c$, $x_{02}=x_{0}-R^{2} / c, \xi=c / R \geq 1$. Углы $\theta, \theta_{1}$ и $\theta_{2}$ обозначены на рис. 2, $a$.

Из определенной таким образом функции Эри найдем поля напряжений $\sigma_{x_{0} x_{0}}, \sigma_{y_{0} y_{0}}, \sigma_{x_{0} y_{0}}$ по известным формулам [68]

$$
\sigma_{x_{0} x_{0}}=\frac{\partial^{2} \psi}{\partial y_{0}^{2}}, \quad \sigma_{y_{0} y_{0}}=\frac{\partial^{2} \psi}{\partial x_{0}^{2}}, \quad \sigma_{x_{0} y_{0}}=-\frac{\partial^{2} \psi}{\partial x_{0} \partial y_{0}} .
$$

Выполнив дифференцирование, получаем:

$$
\begin{aligned}
& \sigma_{x_{0} x_{0}}=\frac{G b_{x_{0}}}{4 \pi(1-v)}\left\{-2\left(\frac{y_{0}}{r_{1}^{2}}+\frac{2 x_{01}^{2} y_{0}}{r_{1}^{4}}\right)+2\left(\frac{y_{0}}{r_{2}^{2}}+\frac{2 x_{02}^{2} y_{0}}{r_{2}^{4}}\right.\right. \\
& \left.-\frac{y_{0}}{r^{2}}+\frac{2 x_{0}^{2} y_{0}}{r^{4}}\right)-\frac{\left(\xi^{2}-1\right) R}{\xi^{3}}\left[\frac{4 x_{02} y_{0}}{r_{2}^{4}}-\frac{16 x_{02}^{3} y_{0}}{r_{2}^{6}}\right. \\
& \left.\left.-\frac{\left(\xi^{2}-1\right) R}{\xi}\left(\frac{2 y_{0}}{r_{2}^{4}}-\frac{8 x_{02}^{2} y_{0}}{r_{2}^{6}}\right)\right]-R^{2}\left(\frac{2 y_{0}}{r^{4}}-\frac{8 x_{0}^{2} y_{0}}{r^{6}}\right)\right\} \\
& +\frac{G b_{y_{0}}}{4 \pi(1-v)}\left\{2\left(-\frac{x_{01}}{r_{1}^{2}}+\frac{2 x_{01}^{3}}{r_{1}^{4}}\right)-2\left(-\frac{x_{02}}{r_{2}^{2}}+\frac{2 x_{02}^{3}}{r_{2}^{4}}\right.\right. \\
& \left.+\frac{x_{0}}{r^{2}}+\frac{2 x_{0}^{3}}{r^{4}}\right)+\frac{\left(\xi^{2}-1\right) R}{\xi^{3}}\left[2 \xi^{2}\left(\frac{1}{r_{2}^{2}}+\frac{2 x_{02}^{2}}{r_{2}^{4}}\right)-\frac{12 x_{02}^{3}}{r_{2}^{4}}\right. \\
& \left.+\frac{16 x_{02}^{4}}{r_{2}^{6}}+\frac{\left(\xi^{2}-1\right) R}{\xi}\left(\frac{6 x_{02}}{r_{2}^{4}}-\frac{8 x_{02}^{3}}{r_{2}^{6}}\right)\right]-2\left(\xi^{2}-1\right) \\
& \left.\times \frac{R}{\xi}\left(\frac{1}{r^{2}}+\frac{2 x_{0}^{2}}{r^{4}}\right)+R^{2}\left(\frac{6 x_{0}}{r^{4}}-\frac{8 x_{0}^{3}}{r^{6}}\right)\right\}, \\
& \sigma_{y_{0} y_{0}}=\frac{G b_{x_{0}}}{4 \pi(1-v)}\left\{-2\left(\frac{y_{0}}{r_{1}^{2}}-\frac{2 x_{01}^{2} y_{0}}{r_{1}^{4}}\right)+2\left(\frac{y_{0}}{r_{2}^{2}}-\frac{2 x_{02}^{2} y_{0}}{r_{2}^{4}}\right)\right. \\
& -\left(\frac{y_{0}}{r^{2}}-\frac{2 x_{0}^{2} y_{0}}{r^{4}}\right)-\frac{\left(\xi^{2}-1\right) R}{\xi^{3}}\left[-\frac{12 x_{02} y_{0}}{r_{2}^{4}}+\frac{16 x_{02}^{3} y_{0}}{r_{2}^{6}}\right. \\
& \left.\left.+\frac{\left(\xi^{2}-1\right) R}{\xi}\left(\frac{2 y_{0}}{r_{2}^{4}}-\frac{8 x_{02}^{2} y_{0}}{r_{2}^{6}}\right)\right]+R^{2}\left(\frac{2 y_{0}}{r^{4}}+\frac{8 x_{0}^{2} y_{0}}{r^{6}}\right)\right\} \\
& +\frac{G b_{y_{0}}}{4 \pi(1-v)}\left\{2\left(-\frac{3 x_{01}}{r_{1}^{2}}-\frac{2 x_{01}^{3}}{r_{1}^{4}}\right)-2\left(\frac{3 x_{02}}{r_{2}^{2}}-\frac{2 x_{02}^{3}}{r_{2}^{4}}\right.\right. \\
& \left.-\frac{3 x_{0}}{r^{2}}-\frac{2 x_{0}^{3}}{r^{4}}\right)-\frac{\left(\xi^{2}-1\right) R}{\xi^{3}}\left[2 \xi^{2}\left(\frac{1}{r_{2}^{2}}-\frac{2 x_{02}^{2}}{r_{2}^{4}}\right)-\frac{4}{r_{2}^{2}}\right. \\
& \left.+\frac{20 x_{02}^{2}}{r_{2}^{4}}-\frac{16 x_{02}^{4}}{r_{2}^{6}}+\frac{\left(\xi^{2}-1\right) R}{\xi}\left(-\frac{6 x_{02}}{r_{2}^{4}}+\frac{8 x_{02}^{3}}{r_{2}^{6}}\right)\right] \\
& \left.-2\left(\xi^{2}-1\right) \frac{R}{\xi}\left(\frac{1}{r^{2}}-\frac{2 x_{0}^{2}}{r^{4}}\right)+R^{2}\left(-\frac{6 x_{0}}{r^{4}}+\frac{8 x_{0}^{3}}{r^{6}}\right)\right\}
\end{aligned}
$$




$$
\begin{gathered}
\sigma_{x_{0} y_{0}}=\frac{G b_{x_{0}}}{4 \pi(1-v)}\left\{-2\left(\frac{x_{01}}{r_{1}^{2}}-\frac{2 x_{01}^{3}}{r_{1}^{4}}\right)+2\left(\frac{x_{02}}{r_{2}^{2}}-\frac{2 x_{02}^{3}}{r_{2}^{4}}\right.\right. \\
\left.-\frac{x_{0}}{r^{2}}-\frac{2 x_{0}^{3}}{r^{4}}\right)-\frac{\left(\xi^{2}-1\right) R}{\xi^{3}}\left[\frac{2}{r_{2}^{2}}-\frac{16 x_{02}^{2}}{r_{2}^{4}}+\frac{16 x_{02}^{4}}{r_{2}^{6}}\right. \\
\left.\left.+\frac{\left(\xi^{2}-1\right) R}{\xi}\left(\frac{6 x_{02}}{r_{2}^{4}}-\frac{8 x_{02}^{3}}{r_{2}^{6}}\right)\right]+R^{2}\left(\frac{6 x_{0}}{r^{4}}-\frac{8 x_{0}^{3}}{r^{6}}\right)\right\} \\
+\frac{G b_{y_{0}}}{4 \pi(1-v)}\left\{2\left(-\frac{y_{0}}{r_{1}^{2}}+\frac{2 x_{01}^{2} y_{0}}{r_{1}^{4}}\right)+2\left(\frac{y_{0}}{r_{2}^{2}}-\frac{2 x_{02}^{2} y_{0}}{r_{2}^{4}}\right.\right. \\
\left.-\frac{y_{0}}{r^{2}}+\frac{2 x_{0}^{2} y_{0}}{r^{4}}\right)+\frac{\left(\xi^{2}-1\right) R}{\xi^{3}}\left[2 \xi^{2} \frac{2 x_{02} y_{0}}{r_{2}^{4}}-\frac{8 x_{02} y_{0}}{r_{2}^{4}}\right. \\
\left.+\frac{16 x_{02}^{3} y_{0}}{r_{2}^{6}}+\frac{\left(\xi^{2}-1\right) R}{\xi}\left(\frac{2 y_{0}}{r_{2}^{4}}-\frac{8 x_{02}^{2} y_{0}}{r_{2}^{6}}\right)\right] \\
\left.-2\left(\xi^{2}-1\right) \frac{R}{\xi} \frac{2 x_{0} y_{0}}{r^{4}}+R^{2}\left(\frac{2 y_{0}}{r^{4}}-\frac{8 x_{0}^{2} y_{0}}{r^{6}}\right)\right\} .
\end{gathered}
$$

Теперь, когда известны напряжения в произвольной точке $K$ в системе координат $\left(x_{0}, y_{0}, z_{0}\right)$, связанной с положением дислокации относительно центра поры, остается найти сдвиговое напряжение в плоскости скольжения этой дислокации в исходной системе координат $(x, y, z)$. Для этого воспользуемся известной формулой преобразования компонент тензора напряжений при повороте системы координат на угол $\varphi[68]$

$$
\sigma_{x y}=-\frac{\sigma_{x_{0} x_{0}}-\sigma_{y_{0} y_{0}}}{2} \sin 2 \varphi+\sigma_{x_{0} y_{0}} \cos 2 \varphi
$$

с учетом преобразования координат $x_{0}=x \cos \varphi+$ $+y \sin \varphi, y_{0}=-x \sin \varphi+y \cos \varphi, z_{0}=z$ и компонент вектора Бюргерса $b_{x_{0}}=b_{x} \cos \varphi, b_{y_{0}}=-b_{x} \sin \varphi$ при $b_{x}=b$.

Получая таким образом напряжение $\sigma_{x y}$ и вычитая из него напряжение $\sigma_{x y}^{\infty}$ краевой дислокации в упругооднородной бесконечной среде в отсутствие поры [69], находим напряжение изображения $\sigma_{x y}^{(h)}$, действующее на дислокацию со стороны поры

$$
\sigma_{x y}^{(h)}=\sigma_{x y}-\sigma_{x y}^{\infty} .
$$

Тогда сила изображения, действующая на $i$-ю дислокацию, определяется как

$$
F_{h i}=b \sigma_{x y}^{(h)}\left(x_{i}, y_{i}\right) .
$$

Рассмотрим теперь суммарную силу, действующую на дислокацию со стороны других дислокаций в присутствие поры. Если в рассматриваемой области находится $N$ дислокаций, то силу $F_{i j}$, действующую на $i$-ю дислокацию со стороны $j$-й дислокации, с учетом экранировки упругого поля последней свободной поверхностью поры

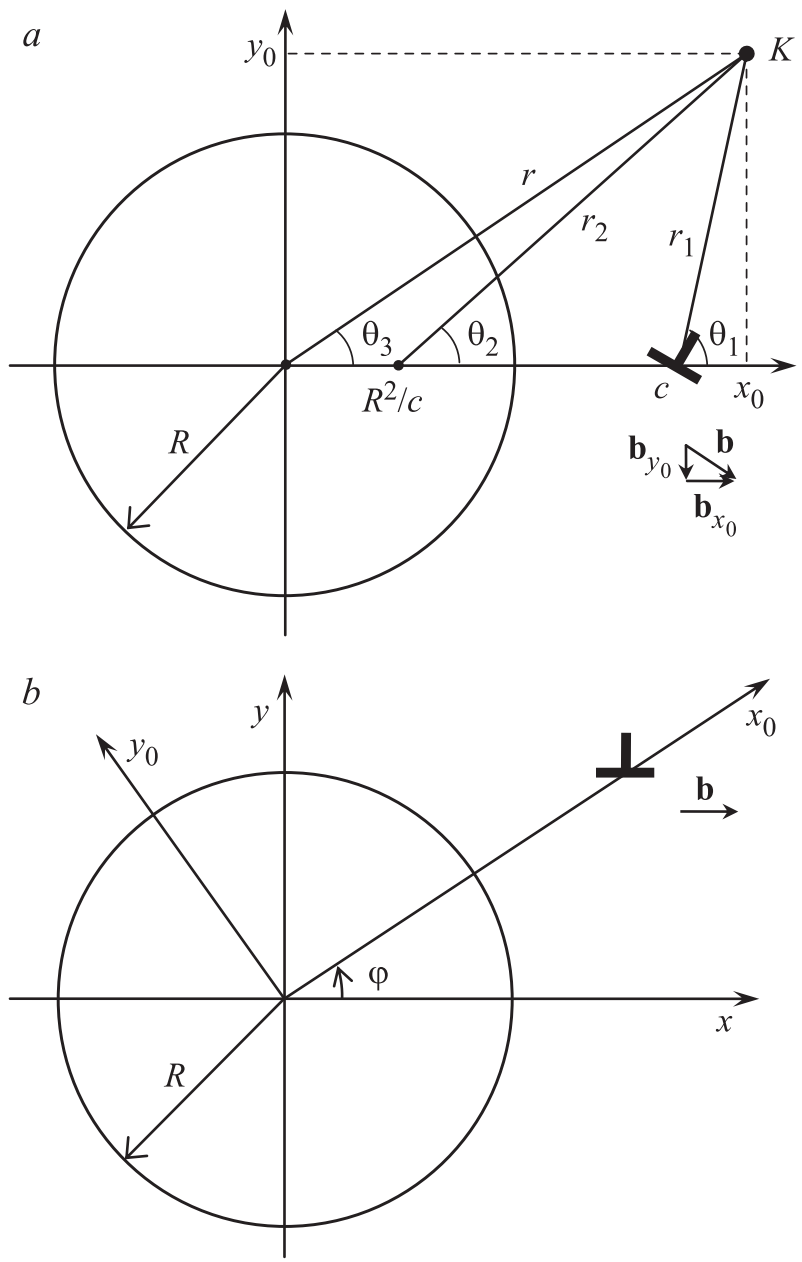

Рис. 2. Положение и ориентация краевой дислокации вблизи поры в повернутой $(a)$ и исходной $(b)$ системах координат, используемых при расчете силы изображения, действующей на дислокацию со стороны поры.

можно записать как

$$
F_{i j}=b \sigma_{x y}\left(x_{i}-x_{j}, y_{i}-y_{j}\right),
$$

где $\sigma_{x y}\left(x_{i}-x_{j}, y_{i}-y_{j}\right)$ - сдвиговое напряжение, создаваемое $j$-й дислокацией, расположенной в точке $\left(x_{j}, y_{j}\right)$, и действующее в точке залегания $i$-й дислокации $\left(x_{i}, y_{i}\right)$. Оно получается из формул (12)-(15) заменой $x$ на $x_{i}-x_{j}$ и $y$ на $y_{i}-y_{j}$.

Таким образом, все слагаемые суммарной силы (2), действующей на произвольную дислокацию вблизи поры, определены.

\section{3. Результаты}

Компьютерное моделирование динамики дискретного дислокационного ансамбля вблизи поры проводилось путем численного интегрирования $N$ уравнений движения вида (1), в правой части которых стоит сила (2). При этом использовался компьютерный код, 
Характеристики дислокационной структуры в зависимости от размера поры

\begin{tabular}{c|c|c|c|c|c}
\hline $\begin{array}{c}\text { Радиус } \\
\text { поры, } \\
\mathrm{nm}\end{array}$ & $\begin{array}{c}\text { Отношение площади } \\
\text { поры к площади } \\
\text { области моделирования }\end{array}$ & $\begin{array}{c}\text { Количество } \\
\text { аннигилировавших } \\
\text { дислокаций }\end{array}$ & $\begin{array}{c}\text { Количество } \\
\text { дислокаций, } \\
\text { поглощенных порой }\end{array}$ & $\begin{array}{c}\text { Количество дислокаций, } \\
\text { вышедших за пределы } \\
\text { области моделирования }\end{array}$ & $\begin{array}{c}\text { Конечная плотность } \\
\text { дислокаций в области } \\
\text { моделирования, ст }{ }^{-2}\end{array}$ \\
\hline 1 & $1.6 \cdot 10^{-4}$ & 48 & 1 & 167 & $1.7 \cdot 10^{11}$ \\
10 & 0.016 & 22 & 29 & 173 & $1.3 \cdot 10^{11}$ \\
30 & 0.141 & 6 & 107 & 129 & $4 \cdot 10^{10}$
\end{tabular}

разработанный в стандартной среде программирования Wolfram Mathematica. Он позволяет получать временные зависимости координаты и скорости каждой дислокации и снабжен специальным интерфейсом визуализации структуры дислокационного ансамбля в виде карты расположения дислокаций в любой момент времени.

Исследование динамики дислокационного ансамбля проводилось внутри двумерной прямоугольной области упруго-изотропной среды с размерами $L \times 2 L$, где $L=100 \mathrm{~nm}$, в центр которой помещалась поpa со свободной поверхностью. Значения упругих модулей системы выбирались для кристалла $\mathrm{GaN}$ в приближении его упругой изотропии согласно данным, полученным в [70]: $G=121 \mathrm{GPa}$, $v=0.26$. Для остальных параметров были выбраны следующие значения: величина вектора Бюргерса $b \approx 2.78 \AA$, плотность $\rho=6150 \mathrm{~kg} \cdot \mathrm{m}^{-3}$, масса дислокации $m=3.1 \cdot 10^{-16} \mathrm{~kg} \cdot \mathrm{m}^{-1}, \beta \approx 7.22 \cdot 10^{-5} \mathrm{~Pa} \cdot \mathrm{s}$. Коэффициент динамического трения определялся по известной [67] формуле $\beta=k_{\mathrm{B}} T B /\left(v_{s}, \Omega\right)$, где $k_{\mathrm{B}}-$ постоянная Больцмана, $T-$ абсолютная температура, $v_{x}-$ скорость звука, $\Omega$ - атомный объем. Все расчеты проводились для $N=250$ дислокаций, вероятность появления которых на экране в определенный момент времени и с определенной координатой по оси $y$ соответствовала равномерному распределению. Каждая дислокация начинала свое движение с линий $x= \pm x_{\text {in }}= \pm 120 \mathrm{~nm}$ за пределами области моделирования. Моделирование проводилось в течение времени $t=10 \mathrm{~ns}$ при значении растягивающего внешнего напряжения $\sigma=10 \mathrm{GPa}$.

Примеры результатов проведенных расчетов представлены на рис. 3 в виде карт конечного распределения ПД вблизи поры. Здесь рассмотрены три случая формирования дислокационной структуры при разных значениях радиуса поры: $R=1 \mathrm{~nm}$ (рис. $3, a$ ), $10 \mathrm{~nm}$ (рис. $3, b)$ и $30 \mathrm{~nm}$ (рис. $3, c)$.

В первом случае (рис. 3,a) пора практически не влияет на формирование дислокационной структуры. Большая часть (167 ПД) за $10 \mathrm{~ns}$ пересекла область моделирования и вышла за ее пределы, меньшая часть (48 ПД) за это время аннигилировала, одна попала в пору и была ею захвачена, а остальные (17 положительных и 17 отрицательных ПД) сформировали устойчивые равновесные дипольные, квадрупольные и мультипольные конфигурации.
Во втором случае (рис. 3,b) наличие поры привело к заметным количественным изменениям в структуре ансамбля ПД. Хотя их большая часть (173 ПД) попрежнему пересекла область моделирования и вышла за ее пределы, и достаточно большое количество (22 ПД) аннигилировало, однако вторая по численности часть ансамбля (29 ПД) оказалась поглощена порой, и только оставшиеся 13 положительных и 13 отрицательных ПД остались в области моделирования вне поры.

В третьем случае (рис. 3,c) пора относительно большого размера поглотила почти половину (107) ПД.
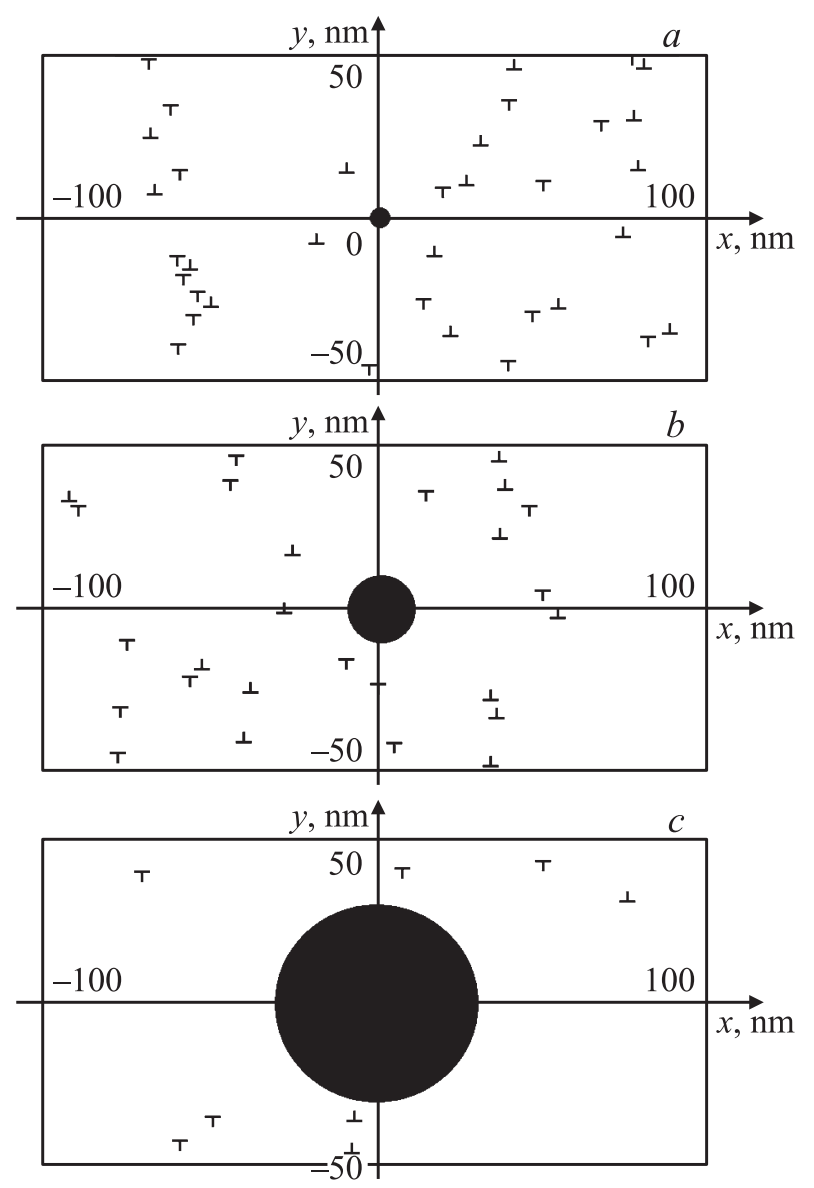

Рис. 3. Карты распределения проникающих дислокаций вблизи цилиндрической поры радиусом $1(1), 10(b)$ и $30 \mathrm{~nm}(c)$ в области моделирования с размерами $200 \times 100 \mathrm{~nm}$, к которой приложено однородное одноосное растягивающее напряжение $10 \mathrm{GPa} \mathrm{под} \mathrm{углом} 45^{\circ}$ к оси $x$. 
Часть (129) ПД прошла мимо поры и вышла за пределы области моделирования, 6 ПД аннигилировали, и всего 8 ПД остались в области моделирования вне поры.

Количественные показатели развития дислокационной структуры вблизи пор за отведенное время $10 \mathrm{~ns}$ под действием растягивающего напряжения $10 \mathrm{GPa}$ сведены в таблице.

\section{4. Заключение}

Проведенное компьютерное моделирование дискретной динамики ПД показало, что создание пор является эффективным способом контроля плотности ПД, генерируемых в процессе релаксации напряжений несоответствия в эпитаксиальных слоях $\mathrm{GaN}$. Полученные результаты говорят о том, что увеличение доли поверхности пор на поверхности слоя до $2 \%$ должно приводить к снижению конечной плотности ПД примерно в 1.5 раза, а увеличение этой доли до $15 \%$ - примерно в 4.5 раза.

\section{Список литературы}

[1] T. Paskova, D.A. Hanser, K.R. Evans. Proc. IEEE 98, 1324 (2010).

[2] S. Nakamura, M.R. Krames. Proc. IEEE 101, 2211 (2013).

[3] J. Millán, P. Godignon, X. Perpiñá, A. Pérez-Tomás, J. Rebollo. IEEE Trans. Power Electronics 29, 2155 (2014).

[4] Z. Alaie, S.M. Nejad, M.N. Yousefi. Mater. Sci. Semicond. Proc. 29, 16 (2015).

[5] S. Fujita. Jpn. J. Appl. Phys. 54, 030101 (2015).

[6] L.Y. Kuritzki, J.S. Speck. MRS Comm. 5, 463 (2015).

[7] T. Matsuoka. Int. J. Optomechatronics 9, 1 (2015).

[8] J. Wang, P. Mulligan, L. Brillson, L.R. Cao. Appl. Phys. Rev. 2, 031102 (2015).

[9] F.A. Marino, N. Faralli, T. Palacios, D.K. Ferry, S.M. Goodnick, M. Saraniti. IEEE Trans. Electron Devices 57, 353 (2010).

[10] S.E. Bennett. Mater. Sci. Technol. 26, 9 (2010).

[11] Y.D. Wang, K.Y. Zang, S.J. Chua, S. Tripathy, C.G. Fonstad, P. Chen. Appl. Phys. Lett. 87, 251915 (2005).

[12] H. Hartono, C.B. Soh, S.Y. Chow, S.J. Chua, E.A. Fitzgerald. Appl. Phys. Lett. 9017191 (2007).

[13] D.H. Lee, J.J. Jang, B.H. Kong, H.K. Cho, O. Nam. Jpn. J. Appl. Phys. Part1. 49, 058001 (2010).

[14] M.G. Mynbaeva, A.E. Nikolaev, A.A. Sitnikova, K.D. Mynbaev. Cryst. Eng. Commun. 15, 3640 (2013).

[15] A.E. Romanov, W. Pompe, G.E. Beltz, J.S. Speck. Appl. Phys. Lett. 69, 3342 (1996).

[16] V.E. Bougrov, M.A. Odnoblyudov, A.E. Romanov, T. Lang, O.V. Konstantinov. Phys. Status Solidi A 203, R25 (2006).

[17] D.M. Artemiev, T.S. Orlova, V.E. Bougrov, M.A. Odnoblyudov, A.E. Romanov. AIP Conf. Proc. 1583, 310 (2014).

[18] D.M. Artemiev, T.S. Orlova, V.E. Bougrov, M.A. Odnoblyudov, A.E. Romanov. J. Electron. Mater. 44, 1287 (2015).

[19] J. Dundurs, T. Mura. J. Mech. Phys. Solids 12, 177 (1964).

[20] E. Smith. Int. J. Eng. Sci. 6, 129 (1968).

[21] J. Dundurs. In: Mathematical Theory of Dislocations / Ed. T. Mura. ASME, N.Y. (1969). P. 70.

[22] G.P. Sendeckyj. Phys. Statis Solidi A 3, 529 (1970).

[23] W.G. Wolfer, W.J. Drugan. Phil. Mag. A 57, 923 (1988).
[24] V.A. Lubarda. J. Elasticity 52, 289 (1999).

[25] M.Yu. Gutkin, A.G. Sheinerman. Phys. Statis Solidi B 231, 356 (2002).

[26] V.A. Lubarda, X. Markenscoff. Mater. Sci. Eng. A 349, 327 (2003).

[27] V.A. Lubarda, M.S. Schneider, D.H. Kalantar, B.R. Remington, M.A. Meyers. Acta Mater. 52, 1397 (2004).

[28] Q.H. Fang, Y.W. Liu. Phys. Status Solidi B 243, R28 (2006).

[29] Q.H. Fang, B. Li, Y.W. Liu. Phys. Status Solidi B 244, 2576 (2007).

[30] М.Ю. Гуткин, А.Г. Шейнерман. ФТТ 49, 1595 (2007).

[31] M.Yu. Gutkin, A.G. Sheinerman, M.A. Smirnov. Mech. Mater. 41, 905 (2009).

[32] M.Yu. Gutkin, A.G. Sheinerman, M.A. Smirnov, V.G. Kohn, T.S. Argunova, J.H. Je, J.W. Jung. Appl. Phys. Lett. 93, 151905 (2008).

[33] V.A. Lubarda. Int. J. Plasticity 27, 181 (2011).

[34] V.A. Lubarda. Int. J. Solids Structures 48, 648 (2011).

[35] S.S. Moeini-Ardakani, M.Yu. Gutkin, H.M. Shodja. Scripta Mater. 64, 709 (2011).

[36] H.M. Shodja, M.Yu. Gutkin, S.S. Moeini-Ardakani. Phys. Status Solidi B 248, 1437 (2011).

[37] A.L. Kolesnikova, M.Yu. Gutkin, S.A. Krasnitckii, A.E. Romanov. Int. J. Solids Structures 50, 1839 (2013).

[38] L. Wang, J. Zhou, D. Hui, S. Zhang. Int. J. Mech. Sci. 79, 168 (2014).

[39] R.J. Amodeo, N.M. Ghoniem. Phys. Rev. B 41, 6958 (1990).

[40] R.J. Amodeo, N.M. Ghoniem. Phys. Rev. B 41, 6968 (1990).

[41] E. van der Giessen, A. Needleman. Mod. Simul. Mater. Sci. Eng. 3, 689 (1995).

[42] S.S. Quek, Z. Wu, Y.W. Zhang, D.J. Srolovitz. Acta Mater. 75, 92 (2014).

[43] S.S. Quek, Z.H. Chooi, Z. Wu, Y.W. Zhang, D.J. Srolovitz. J. Mech. Phys. Solids 88, 252 (2016).

[44] M.Yu. Gutkin, K.N. Mikaelyan, A.E. Romanov, P. Klimanek. Phys. Status Solidi A 193, 35 (2002).

[45] К.Н. Микаелян, М. Seefeldt, М.Ю. Гуткин, P. Klimanek, А.Е. Романов. ФТТ 45, 2002 (2003).

[46] S.V. Bobylev, M.Yu. Gutkin, I.A. Ovid'ko. Acta Mater. 52, 3793 (2004).

[47] С.В. Бобылев, М.Ю. Гуткин, И.А. Овидько, ФТТ 46, 2053 (2004).

[48] Г.Ф. Сарафанов, В.Н. Перевезенцев. Письма в ЖТФ 33, 9 , 87 (2007).

[49] Г.Ф. Сарафанов, В.Н. Перевезенцев. Вопросы материаловедения 49, 1, 5 (2007).

[50] Г.Ф. Сарафанов. ФТТ 50, 1793 (2008).

[51] O. Dmitrieva, J.V. Svirina, E. Demir, D. Raabe. Mod. Simul. Mater. Sci. Eng. 18, 085011 (2010).

[52] Yu.V. Svirina, V.N. Perevezentsev. Tech. Phys. 58, 1224 (2013).

[53] М.Ю. Гуткин, Е.А. Ржавцев. ФТТ 57, 2374 (2015).

[54] E.A. Rzhavtsev, M.Yu. Gutkin. Scripta Mater. 100, 102 (2015).

[55] H.H.M. Cleveringa, E. Van der Giessen, A. Needleman. J. Mech. Phys. Solids 48, 1133 (2000).

[56] M.P. O’Day, W.A. Curtin. J. Mech. Phys. Solids 53, 359 (2005).

[57] T.K. Bhandakkar, A.C. Chng, W.A. Curtin, H. Gao. J. Mech. Phys. Solids 58, 530 (2010).

[58] M. Huang, J. Tong, Z. Li. Int. J. Plasticity 54, 229 (2014). 
[59] S. Liang, M. Huang, Z. Li. Int. J. Solids Structures 56-57, 209 (2015).

[60] M. Huang, Z. Li, C. Wang. Acta Mater. 55, 1387 (2007).

[61] M.I. Hussein, U. Borg, C.F. Niordson, V.S. Deshpande. J. Mech. Phys. Solids 56, 114 (2008).

[62] J. Segurado, J. Llorca. Acta Mater. 57, 1427 (2009).

[63] J. Segurado, J. Llorca. Int. J. Plasticity 26, 806 (2010).

[64] I.A. Ovid'ko, A.G. Sheinerman. Rev. Adv. Mater. Sci. 39, 99 (2014).

[65] I.A. Ovid'ko, A.G. Sheinerman. J. Mater. Sci. 50, 4430 (2015).

[66] Я.В. Конаков, И.А. Овидько, А.Г. Шейнерман. ФТТ 58, 1757 (2016).

[67] U.F. Kocks, A.S. Argon, M.F. Ashby. Prog. Mater. Sci. 19, 73 (1975).

[68] С.П. Тимошенко, Дж. Гудьер. Теория упругости. Наука, М. (1975). 576 c.

[69] Дж. Хирт, Й. Лоте. Теория дислокаций. Атомиздат, М. (1972). $600 \mathrm{c}$.

[70] В.И. Николаев, В.В. Шпейзман, Б.И. Смирнов. ФТТ 42 , 428 (2000). 